\title{
Effet de signaux alternatifs de large amplitude sur la courbe de polarisation d'une interface acier - électrolyte aqueux
}

\author{
I. Ibrahim ${ }^{1}$, H. Takenouti ${ }^{1}$, B. Tribollet ${ }^{1}$, M. Meyer ${ }^{2}$, S. Fontaine ${ }^{3}$ \\ et H.-G. Schoeneich ${ }^{4}$ \\ 1 LISE - UPR 15 du CNRS, Case 133, UPMC, 4 place Jussieu, 75252 Paris Cedex 05, France \\ e-mail : ibrahim77ibrahim@yahoo.fr, ht@ccr; ht@ccr.jussieu.fr ; bernard.tribollet@upmc.fr \\ 2 Gaz de France, 361 avenue du Président Wilson, 93211 Saint-Denis La Plaine, France \\ e-mail : michel.meyer@gazdefrance.com \\ 3 GRTgaz, avenue Ferdinand de Lesseps, 60200 Compiègne, France \\ e-mail : sylvain.fontaine@grtgaz.com \\ 4 EON-Ruhrgas AG, Essen, Allemagne \\ e-mail : hanns-georg.schoeneich@eon-ruhrgas.com
}

Mots-clés :

Protection cathodique; analyse harmonique; courant vagabond; redressement faradique; conduite enterrée

\section{Key words:}

Cathodic protection; harmonic analysis; stray current; faradic rectification; buried pipeline
Résumé - La relation courant - potentiel des systèmes électrochimiques est en général nonlinéaire, ainsi une perturbation alternative de potentiel de grande amplitude induit une réponse en courant contenant une composante continue appelée « redressement faradique ». Nous nous sommes intéressés à l'effet du redressement faradique d'une électrode d'acier au carbone dans un milieu aqueux faiblement minéralisé dans le cadre d'une étude sur la corrosion de conduite de gaz sous protection cathodique et en présence de champ électromagnétique alternatif. Nous avons constaté à l'aide d'un modèle mathématique que l'effet d'un signal alternatif sur la densité de courant moyenne dépend de son amplitude, des coefficients de Tafel, de la résistance d'électrolyte, et du type de processus électrochimique se déroulant à l'interface (activation pure ou cinétique mixte activation-diffusion).

Abstract - Effect of a large amplitude ac signal on the polarization curve of steel aqueous electrolyte interface. Current-potential relationship of electrochemical systems is no-linear. Thus, high amplitude of AC potential perturbation will induce then a current response containing a direct component named as "Faradiac rectification". We are interested about the effect of this Faradaic rectification of carbon steel electrode in simulated natural soil water coming within framework of study about the effect of generated AC electromagnetic field at corrosion of buried pipes at coating defects area. It was shown that the influence of the AC-induced voltage on mean current density strongly depended on its amplitudes, Tafel parameters, electrolyte resistance, and electrochemical processes types which happen at metal interface (activation control or mixed activation-diffusion control).

ouvent les conduites enterrées et les lignes à haute tension se partagent le même territoire dû au «droit de passage », les principales raisons de ce partage sont le coût élevé du droit de passage des conduites et des lignes à haute tension, et les restrictions environnementales imposées par les pouvoirs publiques. Dans ce cas, les pipelines enterrés sont soumis à un haut risque de corrosion par le champ électromagnétique généré par les courants alternatifs AC [1,2].

Malgré la double protection appliquée aux conduites à gaz enterrées, revêtement organique épais et protection cathodique (PC), lorsque ces conduites se trouvent près d'une installation électrique à haute tension, la corrosion peut avoir lieu à la surface du métal au regard du défaut de revêtement de la canalisation.

L'interface de la conduite est perturbée autour de son potentiel de protection cathodique par une tension alternative externe, alors un flux permanent de charge et de matière apparaît. Ceci est dû à des réactions électrochimiques qui induisent un transfert de charge et des gradients de potentiels chimiques et électriques qui provoquent le transport des espèces réagissantes. 
Le phénomène de la corrosion induite par courant alternatif «corrosion $A C$ », peut s'expliquer par deux approches : 1'apparition d'une composante continue due à la non-linéarité des signaux (redressement faradique) et le passage périodique de l'interface dans le domaine anodique [3].

Nous nous sommes intéressés dans ce travail à l'étude de l'effet du redressement faradique sur la courbe de polarisation, c'està-dire à la modification par un signal alternatif en potentiel de grande amplitude de la caractéristique courant - potentiel.

Nous avons présenté précédemment [4] l'effet du redressement faradique sur un processus anodique contrôlé par l'énergie d'activation, c'est-à-dire pour un courant obéissant à une loi exponentielle du potentiel.

Après avoir décrit brièvement l'effet du champ électromagnétique sur les conduites enterrées, nous considérons l'aspect mathématique de l'analyse des harmoniques supérieures. Pour cela, nous présentons l'effet d'une perturbation AC de différentes amplitudes sur un processus cathodique contrôlé par une cinétique mixte (activationdiffusion) en utilisant une électrode d'or comme modèle de l'interface électrochimique. Les résultats obtenus avec une électrode d'acier au carbone dans un milieu aqueux faiblement minéralisé seront enfin analysés.

\section{Principe}

\section{Courant induit sous effet de signal alternatif}

Lorsqu'un potentiel alternatif induit par exemple par une ligne de chemin de fer ou une ligne à haute tension, voir la figure 1, est présent près d'une conduite, le champ électromagnétique va provoquer un courant alternatif qui passe à travers la surface du métal au regard du défaut de revêtement de cette conduite. Ce courant dépend de plusieurs facteurs [4] :

- géométrie physique de la séparation entre les conducteurs et les pipelines,

- la résistivité du revêtement de la canalisation,

- la longueur de la canalisation située en parallèle avec le système de transmission électrique,

- l'amplitude du flux du courant électrique,

- la fréquence du courant alternatif,
- la nature de système électrique, monoou triphasé,

- la résistivité du sol.

En traduisant les facteurs qui affectent l'amplitude du potentiel induit, on peut définir la force électromotrice induite $E_{\mathrm{fem}}$ selon l'équation suivante [5] :

$$
E_{\mathrm{fem}(\mathrm{V})}=I_{(\mathrm{kA})} \cdot M_{(\mathrm{m} \Omega \mathrm{km})} \cdot \omega \cdot L_{(\mathrm{km})}
$$

où $L$ : longueur du parallélisme entre conduite et lignes HT, $M$ : l'inductance mutuelle, $\omega$ : la pulsation du courant.

Donc, on trouve que la $E_{\mathrm{fem}}$. croit avec la résistivité du sol, la densité du courant, la longueur du parallélisme entre pipe et lignes $\mathrm{HT}$, et la fréquence du courant, et décroit avec la distance.

\section{Corrosion AC}

Dans un travail précédent [4], nous avons étudié l'effet des signaux alternatifs sur un processus anodique contrôlé par l'énergie d'activation en utilisant une diode comme un système modèle. La diode est un modèle simple dont le courant suit une loi en fonction du potentiel similaire à une loi de Tafel. Nous avons alors déterminé théoriquement l'expression qui donne le courant moyen anodique en présence de signal AC.

Dans ce travail nous compléterons la modélisation de la corrosion AC en étudiant l'effet du signal alternatif sur un processus cathodique contrôlé par une cinétique mixte (activation-diffusion) à l'aide d'une électrode d'or comme système modèle.

\section{Activation pure}

Si un processus cathodique sous un potentiel stationnaire $E_{0}$ est soumis à une modulation sinusoïdale $\Delta U \cdot \sin (\omega \cdot t)$ : le potentiel s'écrira [6,7] :

$$
E=E_{0}+\Delta U \cdot \sin (\omega \cdot t) .
$$

Et si ce système suit une loi de Tafel, c'està-dire, si le processus est régi par l'énergie d'activation, le courant s'écrit :

$$
I_{\mathrm{c}}(t)=-k_{\mathrm{c}} \cdot \exp \left[-b_{\mathrm{c}} \cdot\left(E_{0}+\Delta U \sin (\omega \cdot t)\right]\right.
$$

où $b_{\mathrm{c}}$ : le coefficient de Tafel cathodique, $k_{\mathrm{c}}$ : la constante de vitesse de réaction à $E=0$. 


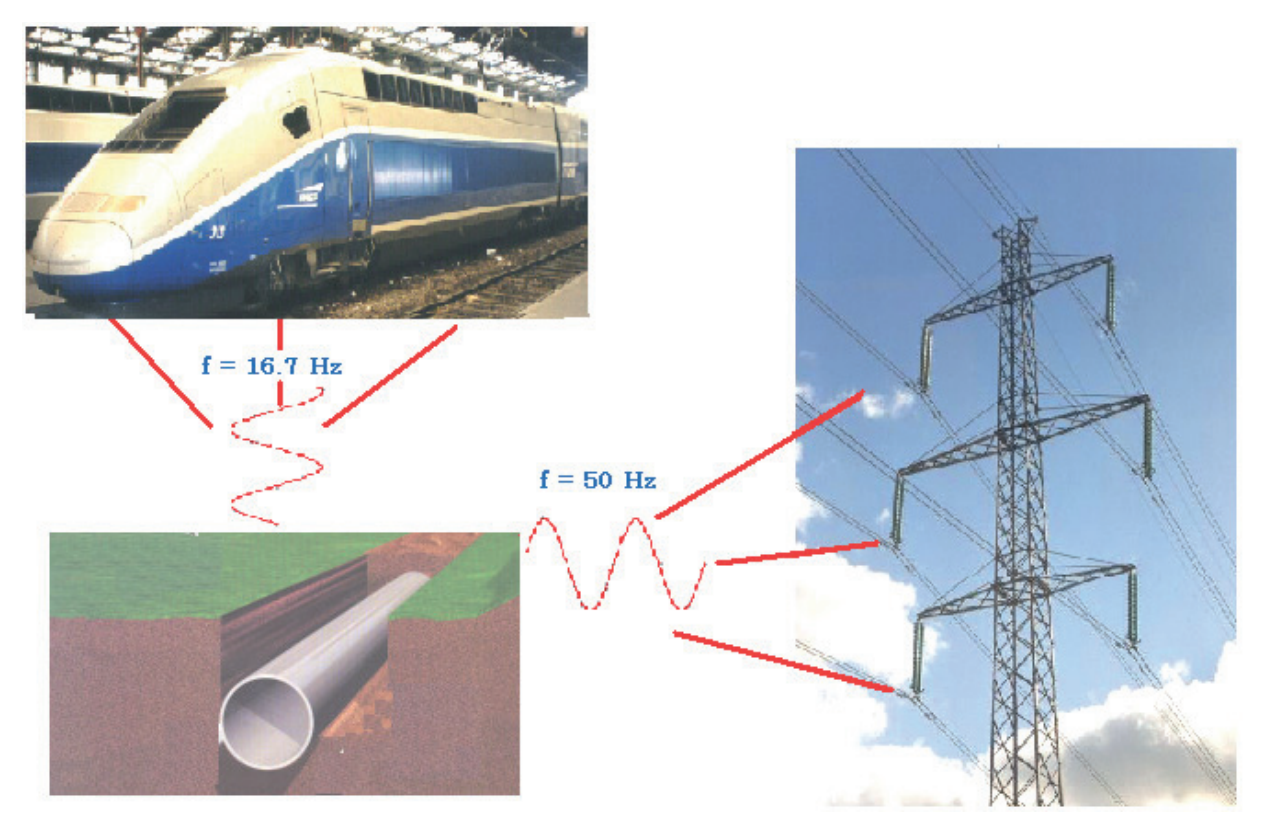

Fig. 1. Effet du champ électromagnétique sur les conduites.

Fig. 1. Electromagnetic field effect on pipelines.

Le développement en série de Taylor de l'expression (3) est :

$$
I_{c}(t)=I_{c, 0} \cdot\left[\begin{array}{l}
1-b_{c} \cdot \Delta U \cdot \sin (\omega \cdot t) \\
+\frac{b_{c}^{2} \cdot \Delta U^{2} \cdot \sin ^{2}(\omega \cdot t)}{2 !} \\
-\frac{b_{c}^{3} \cdot \Delta U^{3} \cdot \sin ^{3}(\omega \cdot t)}{3 !} \\
+\ldots \frac{-b_{c}^{\mathrm{n}} \cdot \Delta U^{\mathrm{n}} \cdot \sin ^{\mathrm{n}}(\omega \cdot t)}{n !}+\ldots
\end{array}\right]
$$

où $I_{\mathrm{c}, 0}=-k_{\mathrm{c}} \cdot \exp \left(-b_{\mathrm{c}} \cdot E_{0}\right)$.

Nous pouvons évaluer le courant cathodique moyen par :

$$
\bar{I}_{c}=\frac{1}{T} \int_{0}^{T} I_{c}(t) \cdot d t
$$

où $T:$ la période du courant alternatif.

Après quelques opérations mathématiques, voir l'annexe sur les opérations d'intégrales, nous obtenons l'expression du courant cathodique moyen en présence de perturbation alternative :

$$
\bar{I}_{c}=-I_{c, 0} \cdot\left(1+\sum \frac{b_{c}^{2 n} \cdot \Delta U^{2 n}}{2^{2 n} \cdot(n !)^{2}}\right)
$$

\section{Cinétique mixte (activation-diffusion)}

Pour une branche cathodique soumis à une cinétique mixte, nous pouvons considérer que le courant cathodique est la somme algébrique du courant de réduction d'oxygène $\left(I_{\mathrm{C}, \mathrm{O}_{2}}\right)$ et du courant de réduction de l'eau $\left(I_{c, \mathrm{H}_{2} \mathrm{O}}\right)$.

$$
\bar{I}_{\mathrm{c}}=\bar{I}_{c, \mathrm{O}_{2}}+\bar{I}_{c, \mathrm{H}_{2} \mathrm{O}}
$$

où $\bar{I}_{\mathrm{c}, \mathrm{O}_{2}}$ peut être écrit suivant l'expression :

$$
\bar{I}_{\mathrm{c}, \mathrm{O}_{2}}=\frac{1}{\left(\bar{I}_{\text {lim }}\right)^{-1}+\left(\bar{I}_{k, \mathrm{O}_{2}}\right)^{-1}} .
$$

Le courant limite de diffusion d'oxygène $\bar{I}_{\text {lim }}$ est donné par l'expression :

$$
\bar{I}_{\lim }=z \cdot F \cdot D_{\mathrm{O}_{2}} \frac{C_{\mathrm{O}_{2, \infty}}}{\delta}
$$

où $D_{\mathrm{O}_{2}}\left(\mathrm{~cm}^{2} . \mathrm{s}^{-1}\right), \delta(\mathrm{cm})$ sont respectivement le coefficient de diffusion et l'épaisseur de la couche de diffusion.

$\bar{I}_{k, \mathrm{O}_{2}}, \bar{I}_{\mathrm{c}, \mathrm{H}_{2} \mathrm{O}}$ : les courants cinétiques de réduction de l'oxygène et de réduction de l'eau sont contrôlés par l'énergie d'activation seule, donc nous pouvons écrire :

$$
\begin{aligned}
\bar{I}_{\mathrm{c}, \mathrm{H}_{2} \mathrm{O}}= & -k_{\mathrm{c}, \mathrm{H}_{2} \mathrm{O}} \cdot \exp \left(-b_{\mathrm{c}, \mathrm{H}_{2} \mathrm{O}} \cdot E_{0}\right) \\
& \cdot\left(1+\sum \frac{b_{c, \mathrm{H}_{2} \mathrm{O}}^{2 \mathrm{O}} \cdot \Delta U_{\mathrm{ac}}^{2 \mathrm{n}}}{2^{2 n} \cdot(n !)^{2}}\right) \\
\bar{I}_{\mathrm{k}, \mathrm{O}_{2}}= & -k_{\mathrm{c}, \mathrm{O}_{2}} \cdot C_{\mathrm{O}_{2}} \cdot \exp \left(-b_{\mathrm{c}, \mathrm{O}_{2}} \cdot E_{0}\right) \\
& \cdot\left(1+\sum \frac{b_{\mathrm{c}, \mathrm{O}_{2}}^{2 \mathrm{n}} \cdot \Delta U_{a c}^{2 \mathrm{n}}}{2^{2 n} \cdot(n !)^{2 !}}\right)
\end{aligned}
$$

où $\mathrm{C}_{\mathrm{O}_{2}}$ est la concentration de l'oxygène dans la solution. Selon notre expérience en utilisant une électrode d'or, nous avons constaté 
(voir la partie expérimentale) que le courant moyen dans le domaine de diffusion $\mathrm{d}^{\prime} \mathrm{O}_{2}$ reste sensiblement constant même sous la perturbation AC.

\section{Effet de résistance d'électrolyte}

Les équations données ci-dessus sont calculées, implicitement, en absence de tout effet de chute ohmique où $E_{\text {off }}$ représente le potentiel interfacial (mesuré expérimentalement au circuit ouvert).

Nous pouvons écrire alors :

$$
U_{\mathrm{on}}=E_{\text {off }}+R \cdot I
$$

où $U_{o n}$ : représente le potentiel imposé en présence de la polarisation donc avec la chute ohmique.

Alors, l'équation du courant peut être récrite par:

$$
\begin{aligned}
I= & k \cdot \exp \left(b \cdot\left(U_{\mathrm{on}}-R \cdot I\right)\right) \Rightarrow \\
& I \cdot \exp (b \cdot R \cdot I)=k \cdot \exp \left(b \cdot U_{\mathrm{on}}\right) .
\end{aligned}
$$

Nous pouvons remarquer que le courant $I$ se trouve deux fois dans cette équation, cependant, il est possible de résoudre cette équation par:

$$
I=\frac{\text { Lambert } \mathrm{W}\left[b \cdot R \cdot k \cdot \exp \left(b \cdot U_{\mathrm{On}}\right)\right]}{b \cdot R} .
$$

Voir l'annexe sur l'équation de Lambert W [8].

En comparaison avec l'équation (13), on peut récrire les équations $(9,10)$ en prenant en compte l'effet de résistance électrolyte :

$$
\begin{array}{r}
\bar{I}_{\mathrm{c}, \mathrm{H}_{2} \mathrm{O}}=\text { Lambert W }\left\{-b_{\mathrm{c}, \mathrm{H}_{2} \mathrm{O}} \cdot R_{\mathrm{e}} \cdot k_{\mathrm{c}, \mathrm{H}_{2} \mathrm{O}}\right. \\
\left.\cdot \exp \left[\left(-b_{\mathrm{c}, \mathrm{H}_{2} \mathrm{O}} \cdot U\right) \cdot\left(1+\sum \frac{b_{\mathrm{c}, \mathrm{H}_{2} \mathrm{O}}^{2 n} \cdot \Delta U^{2 n}}{2^{2 n} \cdot(n !)^{2}}\right)\right]\right\} \\
\times \frac{1}{b_{\mathrm{c}, \mathrm{H}_{2} \mathrm{O}} \cdot R_{\mathrm{e}}}(15) \\
\bar{I}_{k, \mathrm{O}_{2}}=\text { Lambert W }\left\{-b_{\mathrm{C}, \mathrm{O}_{2}} \cdot R_{\mathrm{e}} \cdot k_{\mathrm{C}, \mathrm{O}_{2}} \cdot \mathrm{C}_{\mathrm{O}_{2}}\right. \\
\left.\cdot \exp \left[\left(-b_{\mathrm{c}, \mathrm{O}_{2}} \cdot U\right) \cdot\left(1+\sum \frac{b_{\mathrm{c}, \mathrm{O}_{2}}^{2 n} \cdot \Delta U^{2 n}}{2^{2 n} \cdot(n !)^{2}}\right)\right]\right\} \\
\times \frac{1}{b_{\mathrm{c}, \mathrm{O}_{2}} \cdot R_{\mathrm{e}}} \cdot(16)
\end{array}
$$

Après avoir calculé le courant moyen cathodique sous perturbation $\mathrm{AC}$, on peut maintenant évaluer le courant moyen total $\bar{I}_{T}$ sous

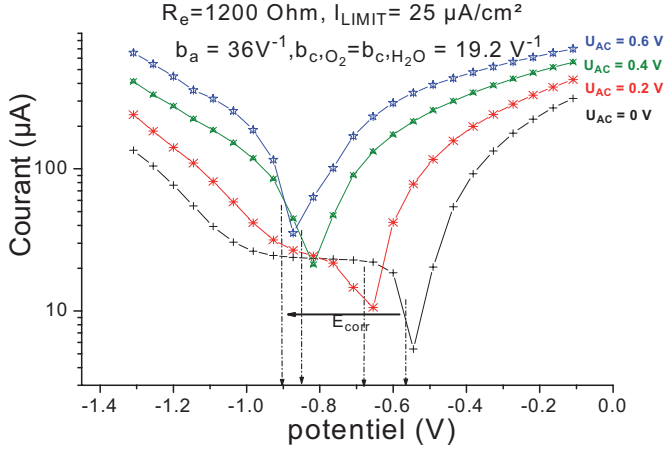

Fig. 2. Modélisation de courbe (I-U) sous modulation AC.

Fig. 2. The modeling of polarization curve under $A C$ perturbation.

AC, où $\bar{I}_{T}$ est la somme du courant moyen anodique et cathodique :

$$
\bar{I}_{T}=\bar{I}_{\mathrm{a}}+\bar{I}_{\mathrm{c}} .
$$

Le courant moyen anodique a été étudié précédemment [3], on peut l'écrire comme :

$$
\begin{array}{r}
\bar{I}_{a}=\operatorname{Lambert} W\left\{b_{\mathrm{a}} \cdot R_{\mathrm{e}} \cdot k_{\mathrm{a}}\right. \\
\left.\cdot \exp \left[\left(b_{\mathrm{a}} \cdot U\right) \cdot\left(1+\sum \frac{b_{a}^{2 n} \cdot \Delta U^{2 n}}{2^{2 n} \cdot(n !)^{2} !}\right)\right]\right\} \\
\times \frac{1}{b_{\mathrm{a}} \cdot R_{\mathrm{e}}}
\end{array}
$$

où $b_{\mathrm{a}}$ : le coefficient de Tafel dans la partie anodique.

Nous avons maintenant toutes les équations nécessaires pour modéliser la courbe de polarisation sous l'effet de l'amplitude AC.

La figure 2 représente l'effet d'une modulation AC de plusieurs amplitudes 0,2; 0,$4 ; 0,6 \mathrm{~V}$ sur la courbe de polarisation pour un système électrochimique correspondant approximativement au système acier $/ \mathrm{NaCl}$ 0,2 g. $\mathrm{L}^{-1}$, pH 5,7 avec les valeurs de paramètres suivantes :

$I_{\text {lim }}=25 \mu \mathrm{A} \cdot \mathrm{cm}^{-2}, b_{\mathrm{a}}=36 \mathrm{~V}^{-1}, b_{\mathrm{c}, \mathrm{O}_{2}}=$ $b_{\mathrm{c}, \mathrm{H}_{2} \mathrm{O}}=19,2 \mathrm{~V}^{-1}, R_{\mathrm{e}}=1200 \mathrm{ohms}$.

Les potentiel standards de $\mathrm{Fe} / \mathrm{Fe}^{2+}, \mathrm{H} / \mathrm{H}^{+}$ et $\mathrm{O}_{2} / \mathrm{OH}^{-}$à $\mathrm{pH} 5.7$ sont respectivement : $-0,82 ;-0,57 ; 0,63 \mathrm{~V}$.

La figure 2 montre que : le potentiel de courant global zéro (potentiel de corrosion $E_{\text {corr }}$ ) en présence de perturbation de tension alternative se déplace vers des valeurs plus négatives. Dans cet exemple le potentiel de corrosion passe de $-0,547 \mathrm{~V}$ en absence de perturbation $\mathrm{AC}$ à $-0,87 \mathrm{~V}$ en présence d'une perturbation AC de $0,6 \mathrm{~V}$. 


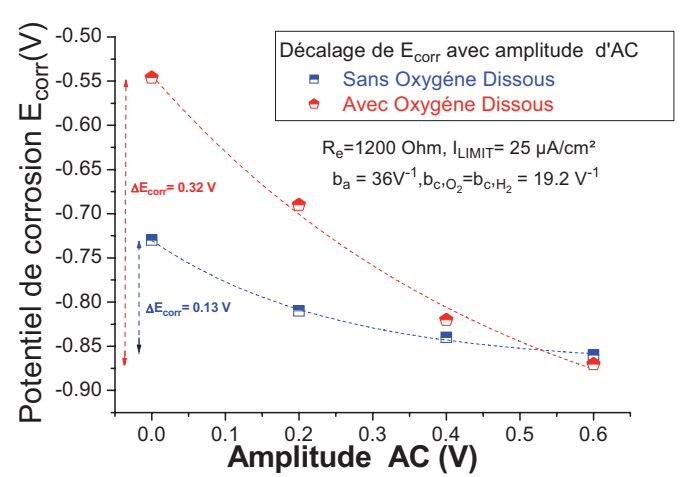

Fig. 3. Décalage de $E_{\text {corr }}$ avec l'amplitude AC pour : 1 - cinétique mixte (activation-diffusion); 2- activation pure.

Fig. 3. Corrosion potential $E_{\text {corr }}$ shift with AC amplitude for : 1- mixed kinetic (activation-diffusion); 2pure kinetic (activation).

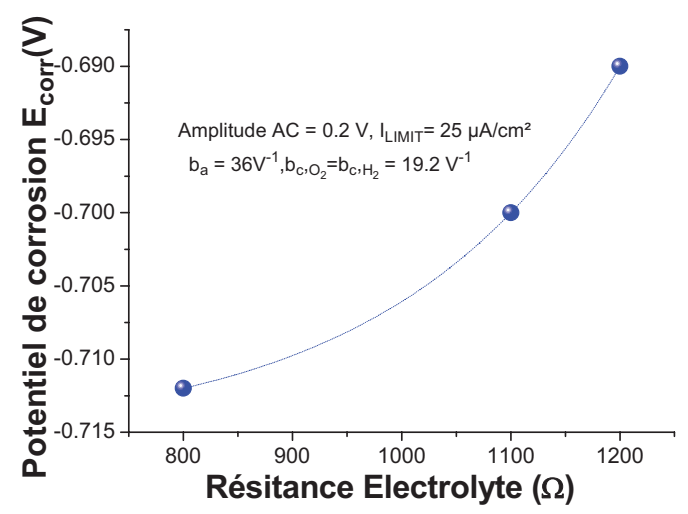

Fig. 4. Effet de résistance d'électrolyte sur le décalage de $E_{\text {corr }}$.

Fig. 4. Electrolyte resistance effect on corrosion potential shift.

Quel est l'effet de d'oxygène dissous sur le décalage du potentiel de corrosion $E_{c o r r}$ ? La figure 3 montre le changement de potentiel de corrosion $E_{c o r r}$ en fonction de l'amplitude du signal AC pour deux types de processus électrochimiques : cinétique mixte (activation-diffusion), voir la figure 2 et activation pure : en absence l'oxygène dissous, $\bar{I}_{C, \mathrm{O}_{2}}$ est égal à zéro, la réaction cathodique est la réduction de l'eau.

Sur cette figure, on peut constater que : le décalage de $E_{\text {corr }}$ est plus important lorsque le système électrochimique est contrôlé par une cinétique mixte.

Maintenant, si on diminue la résistance d'électrolyte $R_{\mathrm{e}}$, le courant moyen augmente et le décalage de $E_{c o r r}$ vers des valeurs plus cathodiques augmente aussi, la figure 4 montre l'effet de la résistance d'électrolyte $R_{\mathrm{e}}$ sur le décalage de potentiel de corrosion $E_{\text {corr }}$ pour une amplitude $\mathrm{AC} U_{\mathrm{ac}}=0,2 \mathrm{~V}$.
Tableau 1. La composition chimique d'eau Evian.

Table 1. Chemical composition of Evian water.

\begin{tabular}{lclc}
\hline Élément & $\mathrm{mg} \mathrm{L}^{-1}$ & Élément & $\mathrm{mg} \mathrm{L}^{-1}$ \\
\hline Calcium & 78 & bicarbonates & 57 \\
Magnésium & 24 & sulfates & 10 \\
Sodium & 5 & Chlorures & 4,5 \\
Potassium & 1 & Nitrates & 3,8 \\
Silice & 13,5 & $\mathrm{pH}$ & 7,4 \\
\hline
\end{tabular}

\section{Conditions expérimentales}

\section{Cellule électrochimique}

Les mesures du courant moyen en présence d'un signal AC sont effectuées en utilisant le dispositif classique à trois électrodes :

\section{Électrode de travail}

L'électrode de travail est un disque en acier au carbone: $(\mathrm{C}=0,1, \mathrm{Mn}=1,34, \mathrm{Si}=0,28$, en $\%$-massique). Les échantillons d'acier, sous forme de barreau cylindrique, ont été fournis par Gaz de France. La surface d'électrode est de $1 \mathrm{~cm}^{2}$.

\section{Électrodes}

La contre électrode est constituée d'un fil de platine. Nous avons utilisé comme référence une électrode au calomel saturée.

\section{Solution électrolytique}

Nous avons utilisé l'eau d'Evian sans purge $\mathrm{d}^{\prime} \mathrm{O}_{2}$, comme représentative de l'eau souterraine naturelle. Le tableau 1 montre sa composition chimique (en $\mathrm{mg} / \mathrm{L}$ ).

\section{Résultats expérimentaux}

\section{Courbe de polarisation en présence de signal AC}

L'objectif de ce travail est d'étudier, de façon globale, comment un signal alternatif en potentiel de grande amplitude modifie la caractéristique courant-potentiel. Afin de connaître l'effet du signal AC, et du niveau de PC sur la courbe de polarisation, plusieurs amplitudes de signal AC et plusieurs valeurs de PC sont appliquées.

Un potentiostat-galvanostat (Solartron SI 1287) a été utilisé pour polariser l'électrode 


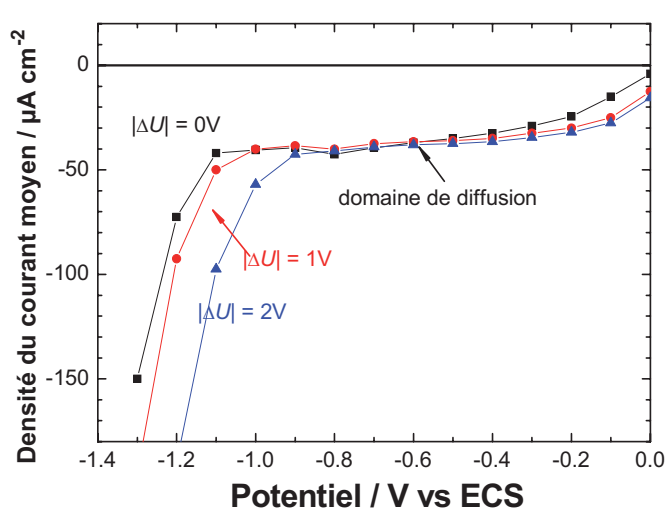

Fig. 5. Effet d'amplitude de potentiel AC sur le courant cathodique moyen pour un système électrode d'or/Eau d'Evian.

Fig. 5. AC amplitude effect on mean cathodic current for gold electrode/Evian water system.

de travail sous régulation de potentiel ou de courant, il est connecté à un analyseur de fonction de transfert (AFT), (Solartron SI 1250) dont le générateur interne envoie une perturbation sinusoïdale au potentiostat.

\section{La courbe (I-E) sous perturbation AC pour une électrode d'or}

L'effet de la perturbation AC de différentes amplitudes sur la réaction cathodique a été étudié avec une électrode $\mathrm{d}$ 'or.

La figure 5 montre la courbe de polarisation d'une électrode plongée dans une solution d'eau Evian, pour trois amplitudes AC. Sur cette figure, on peut constater que le courant moyen dans le domaine de diffusion $d^{\prime} \mathrm{O}_{2}$ reste sensiblement le même. À des potentiels inférieurs à $-1 \mathrm{~V}$, le courant moyen augmente en valeur absolue. À ces potentiels, c'est le dégagement d'hydrogène qui est le processus dominant. Le courant suit de nouveau une loi exponentielle en potentiel.

\section{Courbe (I-E) sous AC (Acier - Eau d'Evian)}

La figure 6 montre les courbes courantpotentiel obtenues avec signal AC sur l'électrode d'acier au carbone dans l'eau d'Evian. Nous avons utilisé l'eau juste après l'ouverture de la bouteille pour minimiser l'effet du $\mathrm{CO}_{2}$ ambiant.

Nous constatons que le courant moyen de protection cathodique en présence de perturbation de tension alternative diminue en valeur absolue. D'autre part, le potentiel à

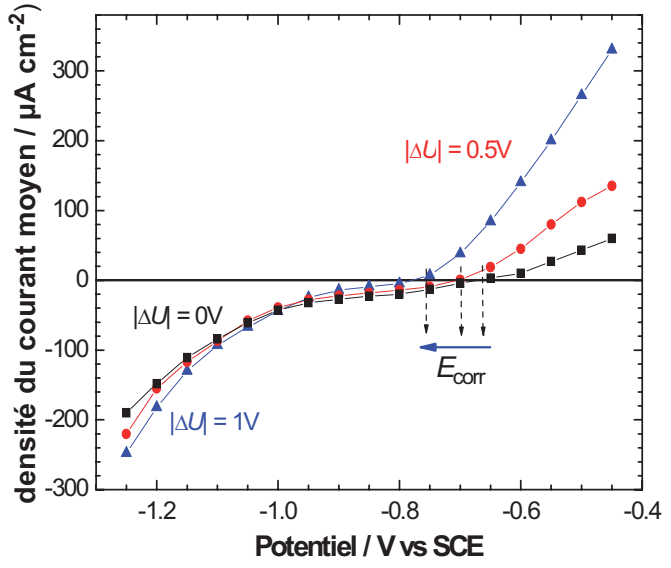

Fig. 6. Courbes (I-E) avec signal AC. Acier/Eau d'Evian. $f=50 \mathrm{~Hz}$.

Fig. 6. (I-E) curves under AC signal. Carbon steel/Evian water. $f=50 \mathrm{~Hz}$.

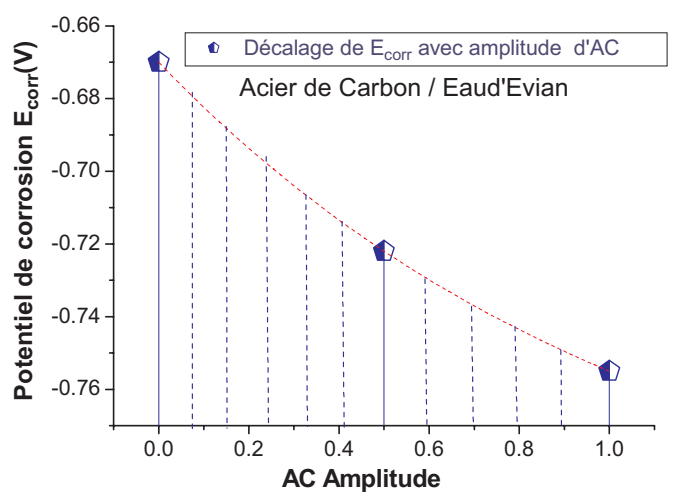

Fig. 7. Le décalage d' $E_{\text {corr }}$ sous l'effet d'amplitude.

Fig. 7. Potential corrosion shift under AC amplitude effect.

courant nul (potentiel de corrosion) se déplace vers des valeurs plus négatives ce qui rend la protection cathodique moins efficace.

La figure 7 montre le décalage du potentiel de corrosion en fonction de l'amplitude de signal AC pour l'eau d'Evian.

\section{Conclusion}

Pour les systèmes électrochimiques nonlinéaires, la réponse d'un courant induit par un signal alternatif de potentiel de grande amplitude induit une composante continue appelée « redressement faradique ».

Les résultats du calcul de simulation et les résultats expérimentaux ont clairement montré que le courant moyen en présence d'une perturbation AC change avec :

- l'amplitude de perturbation alternative $\Delta U_{\mathrm{ac}}$ 
- les coefficients de Tafel $b_{\mathrm{a}}, b_{\mathrm{c}}$

- la résistance d'électrolyte $R_{\mathrm{e}}$

- le type de processus électrochimique se déroulant à l'interface (activation pure ou cinétique mixte activation-diffusion).

\section{Annexe 1}

Le développement en série de Taylor de l'équation (3) est donné par l'équation (4).

À l'aide de l'expression suivante :

$$
\begin{aligned}
\int \sin ^{n}(x) \cdot d x= & -\frac{\sin ^{n-1}(x) \cdot \cos (x)}{n} \\
& +\frac{n-1}{n} \int \sin ^{n-2}(x) \cdot d x
\end{aligned}
$$

Le courant moyen cathodique peut être écrit :

$\bar{I}_{\mathcal{C}}=\frac{1}{T} \int_{0}^{T} I_{\mathcal{c}, 0}\left\{1+\sum_{1}^{n} \frac{b_{c}^{n} \Delta U^{n}}{n !} \sin ^{n}(\omega . t)\right\} . d t \Rightarrow$

$$
\bar{I}_{c}=-\frac{I_{c, 0}}{T}\left\{T+\sum_{1}^{n} \frac{b_{c}^{n} \Delta U^{n}}{n !}\left[\frac{1}{n} \sin ^{n-1}(\omega . t) \cos (\omega . t)\right]_{0}^{T}\right.
$$$$
\left.+\sum_{1}^{n} \frac{b_{c}^{n} \Delta U^{n}}{n !}\left(\frac{n-1}{n} \int_{0}^{T} \sin ^{n-2}(\omega \cdot t) \cdot d t\right)\right\} \text {. }
$$

Le terme : $\left[\frac{1}{n} \sin ^{n-1}(\omega . t) \cos (\omega . t)\right]_{0}^{T}$ est égal à zéro, donc, le courant moyen cathodique peut s'écrire alors :

$$
\begin{aligned}
\bar{I}_{c}=-I_{c, 0}\left(1+\frac{1}{T} \sum_{1}^{n} \frac{b_{c}^{n} \Delta U^{n}}{n !}\right. \\
\\
\left.\quad\left(\frac{n-1}{n} \int_{0}^{T} \sin ^{n-2}(\omega \cdot t) \cdot d t\right)\right) .
\end{aligned}
$$

Quand, $n$ est impair, l'intégrale est zéro :

$$
\begin{aligned}
& \frac{n-1}{n} \int_{0}^{T} \sin ^{n-2}(\omega \cdot t) \cdot d t=\frac{n-1}{n} \times \frac{n-3}{n-2} \\
& \times \frac{n-5}{n-4} \times \ldots . \frac{2}{3} \times \int_{0}^{T} \sin ^{n}(\omega \cdot t) \cdot d t=0 .
\end{aligned}
$$

Alors l'équation du courant moyen cathodique :

$$
\bar{I}_{\mathrm{c}}=-I_{c, 0} \cdot\left(1+\sum \frac{b_{\mathrm{C}}^{2 \mathrm{n}} \cdot \Delta U^{2 \mathrm{n}}}{2 n !} \cdot \alpha_{2 n}\right)
$$

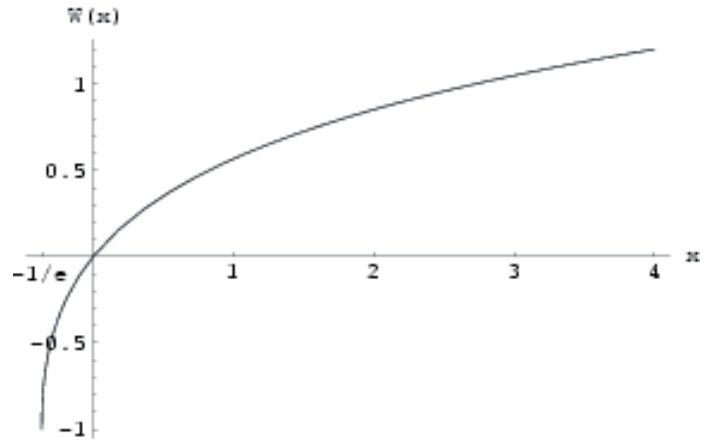

où

$$
\begin{aligned}
\alpha_{2 n}=\frac{2 n-1}{2 n} & \times \frac{2 n-3}{2 n-2} \\
& \times \frac{2 n-5}{2 n-4} \times \ldots \ldots \frac{1}{2}=\frac{2 n !}{2^{2 n} \cdot(n !)^{2}} .
\end{aligned}
$$

En remplaçant $\alpha_{2 n}$ par sa valeur, on obtient l'expression (6).

\section{Annexe 2}

La fonction $\mathbf{W}$ de Lambert, nommée ainsi par Johann Heinrich Lambert, est aussi appelée la fonction Oméga. Elle est la fonction réciproque de $f$ définie :

Pour tout nombre complexe $\omega, f(\omega)=$ $\omega \exp (\omega)$.

Ce qui implique que pour tout nombre complexe $z$, nous avons :

$W(z) \cdot \exp (W(z))=z$

$Y=X \cdot \exp (X) \quad \Leftrightarrow \quad X=W(Y)$.

Puisque la fonction $f \mathrm{n}^{\prime}$ est pas injective, la fonction $W$ est multiforme.

Si nous nous limitons aux arguments réels $x \geq-1 / e$ (ce qui exige $\omega \geq-1$ ) alors il existe une fonction et une seule $W_{0}$ définie, dont la représentation graphique figure ci-dessous.

Nous avons $W_{0}(0)=0$ et $W_{0}(-1 / e)=-1$.

La fonction $W$ de Lambert ne peut pas être exprimée à l'aide de fonctions élémentaires. Elle est utile en combinatoire, par exemple dans l'énumération des arbres. Elle peut être utilisée pour résoudre diverses équations qui comportent des exponentielles et apparaît aussi dans les solutions d'équations différentielles à temps-retardés, telles que $y^{\prime}(t)=a \quad y(t-1)$. 


\section{Références}

[1] W. Bichard, P.E. Bonds, The Effect of overhead AC power lines paralleling ductile iron pipelines, DIPRA Research and technical director, 1999

[2] P.D. Simon, J.T. Schmidt, B.K. Mumme, Dynamic nature of HVAC induced current density on collocated pipelines, NACE, Paper No. 07650

[3] M. Buchler, F. Stalder, H.G. Schöneich, New Electrochemical Method fort he Detection of AC Corrosion, CEOCOR DRESDEN - Sector A, Paper No. 07

[4] I. Ibrahim, B. Tribollet, H. Takenouti, X. Campaignole, S. Fontaine, P. France, H.G. Schoeneich, Étude du redressement faradique par analyses harmoniques, in "Acte $\mathrm{du}$ Forum sur les impédances électrochimiques ", 20 (2006), C2, édité par C. Gabrielli, Paris

[5] M.H. Shwehdi, U.M. Johar, Transmission line EMF interference with buried pipeline:
Essential and cautions, Proceedings of international conference on non-ionizing at UNITEN (ICNIR 2003)

[6] C. Mouychard, Induction des lignes à haute tension sur les canalisations enterrées, Présentation au Rencontre 6, de CEFRACOR, Paris, 4 Déc. 2006

[7] I. Ibrahim, B. Tribollet, C. Drevet, H. Takenouti, X. Campaignole, Effet du courant alternatif sur la corrosion des conduites enterrées sous protection cathodique, E08, CEFRACOR «Protection cathodique», June 2006, Aix en Provence, France

[8] I. Ibrahim, B. Tribollet, H. Takenouti, X. Campaignole, S. Fontaine, P. France, H-G. Schoeneich, Harmonic Analysis Study of the AC Corrosion of Buried Pipelines, Under Cathodic Protection, NACE, Corrosion 2007, Paper No. 07042

[9] Op. cit: Corless et al., Adv. Comput. Math. 5 (1996) 329 